\title{
Biomolecule-directed assembly of nanoscale building blocks studied via lattice Monte Carlo simulation
}

\author{
Ting Chen, Monica H. Lamm, ${ }^{\text {a) }}$ and Sharon C. Glotzer ${ }^{\text {b) }}$ \\ Department of Chemical Engineering, University of Michigan, Ann Arbor, Michigan 48109-2136
}

(Received 7 April 2004; accepted 26 May 2004)

\begin{abstract}
We perform lattice Monte Carlo simulations to study the self-assembly of functionalized inorganic nanoscale building blocks using recognitive biomolecule linkers. We develop a minimal coarse-grained lattice model for the nanoscale building block (NBB) and the recognitive linkers. Using this model, we explore the influence of the size ratio of linker length to NBB diameter on the assembly process and the structural properties of the resulting aggregates, including the spatial distribution of NBBs and aggregate topology. We find the constant-kernel Smoluchowski theory of diffusion-limited cluster-cluster aggregation describes the aggregation kinetics for certain size ratios. (C) 2004 American Institute of Physics. [DOI: 10.1063/1.1774154]
\end{abstract}

\section{INTRODUCTION}

"Bottom-up" assembly of inorganic nanoparticles and supramolecular entities is a promising means for creating advanced materials with superior electronic, optical, and mechanical properties. ${ }^{1,2}$ Synthetic methods continue to improve for creating nanoscale building blocks (NBBs) of uniform size and shape, e.g., nanorods, ${ }^{3,4}$ nanocubes, ${ }^{5}$ and nanoprisms, ${ }^{6}$ and for functionalizing NBBs with organic "handles" for subsequent assembly, ${ }^{70,71}$ e.g., nanoparticles, ${ }^{7-10}$ nanorods, ${ }^{11}$ nanotubes, ${ }^{12-15}$ fullerenes, ${ }^{16}$ and cubic silsesquioxanes. ${ }^{17-20}$ NBBs assembled into ordered structures using biological ${ }^{21-23}$ or synthetic molecules with recognition elements ${ }^{24-26}$ as linkers belong to a novel class of hybrid nanoscale systems that are promising candidates for designing materials and devices with specific properties and functionalities. The use of biological-based linkers provides predictable, specific recognition interactions that can be tailored (e.g., introducing a spacer sequence or adjusting the binding affinity) and reversed (e.g., annealing to avoid kinetically arrested structures) to create designer materials. $^{27,28}$

Several elegant demonstrations of biomolecule-directed assembly have been reported in the literature. Mirkin et al. ${ }^{21}$ demonstrated that by grafting oligonucleotides onto the surface of $13 \mathrm{~nm} \mathrm{Au}$ particles, DNA strands with nucleotide sequences complementary to those attached to the nanoparticles can be used to tether the particles into nanoparticle superlattices. Mirkin and co-workers have since used the strategy of DNA-programmed assembly to construct organized arrangements of nanoparticles of different size ${ }^{29}(8 \mathrm{~nm}$ and $31 \mathrm{~nm}$ Au particles) and different chemical composition ${ }^{7}$ ( $3 \mathrm{~nm} \mathrm{CdSe}$ and $13 \mathrm{~nm} \mathrm{Au} \mathrm{particles).} \mathrm{They} \mathrm{have} \mathrm{also} \mathrm{shown}$ that $\mathrm{Au}$ nanoparticles can be assembled using hybrid DNA/ block copolymer linkers, ${ }^{30}$ thereby creating an opportunity to

\footnotetext{
${ }^{a}$ Current address: Department of Chemical Engineering, Iowa State University, Ames, Iowa 50011-2230.

b) Also at Department of Materials Science and Engineering, University of Michigan, Ann Arbor, Michigan 48109-2136. Author to whom correspondence should be addressed. Electronic mail: sglotzer@umich.edu
}

further tailor the properties of the final NBB/macromolecule assembly through the choice of block copolymer. Dujardin et al. $^{11}$ used DNA-programmed assembly to assemble $50 \times 10 \mathrm{~nm} \mathrm{Au}$ nanorods in solution.

Other types of highly specific biomolecular interactions have been exploited as well. Connolly and Fitzmaurice ${ }^{31}$ used a protein-substrate binding strategy to assemble $16 \mathrm{~nm}$ $\mathrm{Au}$ nanoparticles whereby the nanoparticles are functionalized with a disulfide-biotin analogue and then crosslinked with streptavidin. A similar strategy was used by Li et al. ${ }^{32}$ to assemble iron oxide particles. Shenton et al. ${ }^{33}$ used antibody-antigen recognition motifs to assemble $12 \mathrm{~nm} \mathrm{Au}$ nanoparticles and bimetallic networks of $12 \mathrm{~nm} \mathrm{Au}$ and $\mathrm{Ag}$ particles. Xu et al. ${ }^{9}$ used peptide bond formation to assemble $13 \mathrm{~nm} \mathrm{Au}$ particles. Boal et al. ${ }^{24}$ attached thyminefunctionalized alkanethiols to $2 \mathrm{~nm}$ Au nanoparticles and assembled the particles using diaminotriazine-functionalized polystyrene. The structures observed by Boal et al. showed an interesting dependence on temperature; at $10^{\circ} \mathrm{C}$, small (20-40 nm) spherical aggregates formed a braided structure while at temperatures above and below, larger (100-1000 $\mathrm{nm})$ spherical aggregates formed.

A limited number of systematic, in-depth studies on biomolecule-directed assembly have been undertaken. Storhoff et al. ${ }^{34}$ linked $15 \mathrm{~nm} \mathrm{Au}$ particles functionalized with (alkanethiol)-12 base oligomers using DNA linkers of 24, 48, and 72 base pairs to explore the effect of linker length on the formation and properties of the resulting aggregates. They found that the shortest linker gave the fastest aggregation and yielded the largest aggregates. Li et al. ${ }^{32}$ added streptavidin to biotinylated ferritin/iron oxide particles in ratios of $2: 1$, $4: 1,6: 1$, and $8: 1$ to investigate the effect of linker to particle stoichiometric ratio. They found that a ratio of at least $4: 1$ (streptavidin to ferritin) was needed to induce aggregation and that 6:1 was the optimum stoichiometric ratio for this system. The rate of aggregation increased as concentration increased. Aside from these studies, very little of the vast parameter space of biomolecule-directed assembly has been explored. For example, by adjusting the length, rigidity, and 
specificity of the linkers one might exert control over the spatial distribution of NBBs. In addition, because the highly specific interactions between biomolecule linkers are sensitive to changes in temperature and ionic strength in the surrounding medium, it should be possible to control the assembly process by adjusting the solvent conditions.

Computer simulation is an ideal tool to help identify key variables and efficiently map the parameter space in biomolecule-directed assembly systems, as well as provide insight to the biomolecule-directed assembly process. In this paper, we propose a minimal lattice model for simulating large numbers of NBBs assembled by linkers with specific, recognitive interactions. Using this model, we explore how the size ratio of linker length to NBB diameter affects the aggregation rate and influences structural properties of the resulting aggregates, including the spatial distribution of NBBs and the aggregate topology. Highlights of our results are as follows: By monitoring the number of aggregates in the system versus time, we find that the Smoluchowski theory of diffusion-limited cluster-cluster aggregation can be used to describe the aggregation. For size ratios of linker length to NBB diameter less than one, the rate of aggregation follows classical Smoluchowski kinetics, where the aggregation kernel is assumed to be constant. We demonstrate control over the aggregate structure by adjusting the NBB size and linker length. We also show how the topology of the linker connections between NBBs directly influences the spatial distribution of NBBs.

The remainder of the paper is organized as follows: Section II describes the minimal model for NBBs and biomolecule linkers, and gives a brief overview of the lattice Monte Carlo method. Section III contains results from the simulations, including aggregation rate and structural analysis, and a brief summary and conclusions are given in Sec. IV.

\section{MODEL AND SIMULATION METHOD}

The NBBs and linkers are studied using the bond fluctuation model and algorithm ${ }^{35-37}$ on a simple cubic lattice. Restricting the motion of the NBB and the linkers to the vertices of a finely grained lattice alleviates, to some degree, the computational challenge of simulating large collections of NBBs and linkers assembling into larger scale structures. ${ }^{38}$ The bond fluctuation algorithm is a dynamic Monte Carlo method and has been widely adopted for simulating the formation and dynamics of polymer networks. ${ }^{39-46}$ An endlinked polymer network (where the size of the crosslinks is similar to the size of the monomer in a polymer chain) represents a limiting case of NBB-linker assembly (where the NBB is larger than the monomer in a linker). In the present application of the bond fluctuation model a NBB is modeled as a cube occupying $d \times d \times d$ lattice vertices and a linker is modeled as a series of $L$ connected monomers, where each monomer occupies $2 \times 2 \times 2$ lattice vertices. The allowed bond vectors between two consecutive monomers in a linker are the following: $[2,0,0],[2,1,0],[2,1,1],[2,2,1]$, $[3,0,0]$, and $[3,1,0] .^{36}$

The NBB-linker model presented below is necessarily simple to allow for the simulation of several thousands of NBBs, yet includes sufficient detail to mimic key aspects of

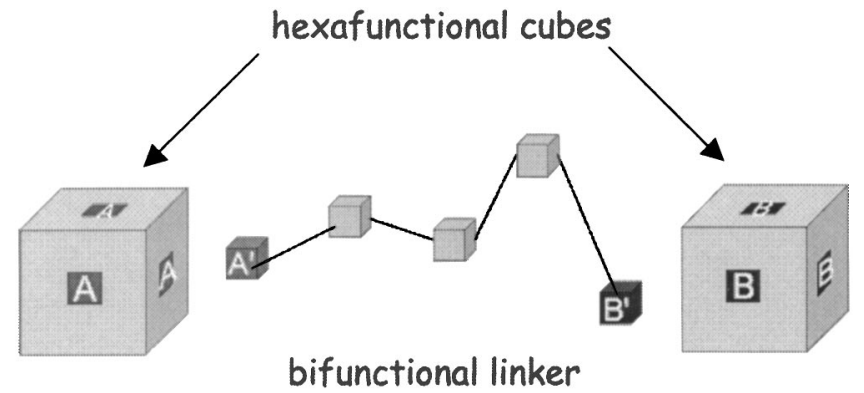

FIG. 1. Illustration of the NBB-linker model used in this work. NBBs occupy $d \times d \times d$ lattice sites; linker monomers occupy $2 \times 2 \times 2$ lattice sites. Reactive sites $A$ or $B$ occupying $2 \times 2$ lattice sites are placed at the six face centers of each NBB. Each linker has one $A^{\prime}$ terminal monomer and one $B^{\prime}$ terminal monomer. Complementary $A-A^{\prime}$ and $B-B^{\prime}$ interactions are implemented as described in the text.

the highly specific interactions utilized in biomoleculedirected assembly of inorganic nanoparticles. A schematic of the model is shown in Fig. 1. Reactive sites, occupying an area of $2 \times 2$ lattice vertices, are placed at the six face centers of each NBB. In principle, reactive sites could be placed on the NBB with any desired pattern and surface coverage. The choice of six reactive sites per NBB for this work coincides with the average number of hybridized DNA linkers per 15.7 $\mathrm{nm}$ Au nanoparticle, as determined experimentally by Demers et $a l .{ }^{47}$ The reactive sites on half of the NBBs are designated as $\mathbf{A}$ sites and the reactive sites on the remaining NBBs are designated as $\mathbf{B}$ sites. To mimic the complementary nature of the interactions, such as one might program into the sequence of a DNA strand, one end of a linker is designated as an $\mathbf{A}^{\prime}$ monomer and the other end of the linker is designated as a $\mathbf{B}^{\prime}$ monomer. When an unreacted linker end comes into nearest neighbor contact with an unreacted complementary NBB face center, a crosslink forms with energy $\epsilon / k T$ $=-100$, where $\epsilon$ is the crosslink bond energy for either an $\mathbf{A A}^{\prime}$ or $\mathbf{B B}^{\prime}$ bond, $k$ is the Boltzmann constant, and $T$ is the system temperature. The value 100 is arbitrarily chosen so that crosslinks, once formed, rarely break unless the temperature is raised. This strong interaction is meant to mimic the effective strength of bonding between two hybridized DNA strands, where cooperativity of many weak hydrogen bonds results in a strong association at low temperature. ${ }^{48}$ To facilitate diffusion of the NBB once linkers are attached to its surface the set of allowed bond vectors between a monomer and a NBB reactive site is increased to include these additional vectors: $[2,2,2],[3,1,1],[3,2,1],[4,0,0] .{ }^{49,50}$

A typical simulation proceeds as follows. First, 1000 NBBs and 3000 linkers are randomly placed on a cubic lattice at a volume fraction $\phi=0.01$. In this paper we consider only a stoichiometric ratio of linkers to NBBs; nonstoichiometric cases will be studied in a separate publication. Here we consider NBBs with diameter $d=4,8$, or 12 (measured in units of lattice vertices) and linkers of length $L=3,5$, or 7 (measured in the number of monomers). The system is then allowed to relax without the attractive interactions between functional sites on nanoparticles and the linker end monomers. The initial, effectively high temperature relaxation is carried out for approximately 1 million Monte Carlo steps 
(MCS), where in one MCS every particle, including nanoparticles and monomers, attempts one move on average. The purpose of this procedure is to ensure the system is wellmixed prior to assembly. Following the relaxation period the specific attractions between NBB reactive sites and linker ends are instituted. During this period we monitor the fraction of unreacted linker ends, the number of aggregates, and the topology of the aggregates as a function of time.

\section{RESULTS}

In this section we describe the results obtained from lattice Monte Carlo simulations of NBBs assembled by linkers with specific interactions. Although there are several factors that may affect the growth of biomolecule-linked NBB aggregates, two critical factors are believed to be (i) the rate of a biomolecule linker binding to a complementary site on the NBB surface and (ii) the rate of a NBB with bound biomolecule linkers attaching to complementary NBBs. ${ }^{34}$ We begin by examining each of these mechanisms, in turn, to characterize the assembly process of the model NBB and biomolecule linker system. We then investigate the structural properties of representative aggregates from each system.

\section{A. Rate of linkers binding to NBBs}

To assess the rate at which linkers bind to NBBs, we monitor the fraction of unreacted linker ends, $x_{f}$, versus simulation time, $t$ (in MCS). ${ }^{51}$ Figure 2 shows the fraction of unreacted linker ends versus MCS for systems with NBBs of diameter $d=4,8$, and 12 , and linkers of length $L=3,5$, and 7. In Fig. 2(a), for the case of NBB with $d=4$, the fraction of unreacted linker ends at early times is lowest for the linker with $L=3$. After approximately 7 million MCS, the fraction of unreacted linker ends decreases most rapidly for the linker with $L=7$. This trend can be explained as follows: In the initial stages of linker to NBB binding, the shortest linker (here, $L=3$ ) will diffuse faster in solution and more readily find the reactive site. However, as the reaction proceeds, linkers bound to the NBB sterically hinder the remaining free linkers from reaching the remaining NBB reactive sites. A longer linker (here, $L=7$ ) has more degrees of freedom and is more likely to find the reaction site on the surface of the NBB. Similar behavior is observed for NBBs with $d=8$ [Fig. 2(b)] and 12 [Fig. 2(c)], where the effect of linker length is more pronounced and the crossover in the fraction of unreacted linker ends occurs at much earlier times of 1 and 1.4 million MCS, respectively. For a given linker length, the NBB with $d=4$ has the lowest fraction of unreacted linker ends, followed by $d=8$ and $d=12$, respectively. The trend toward more complete reaction as the NBB diameter decreases is expected for the model system considered here where the number of reactive sites on each particle is held constant. The number of reactive sites per unit surface area of NBB for $d=4$ is thirteen times higher than for $d=12$. Another reason is that in the simulation we keep the overall volume fraction fixed, so as the diameter of the NBB decreases and hence, the volume fraction occupied by NBBs decreases, the volume fraction occupied by linker ends increases. As a result, the rate and extent of reaction between

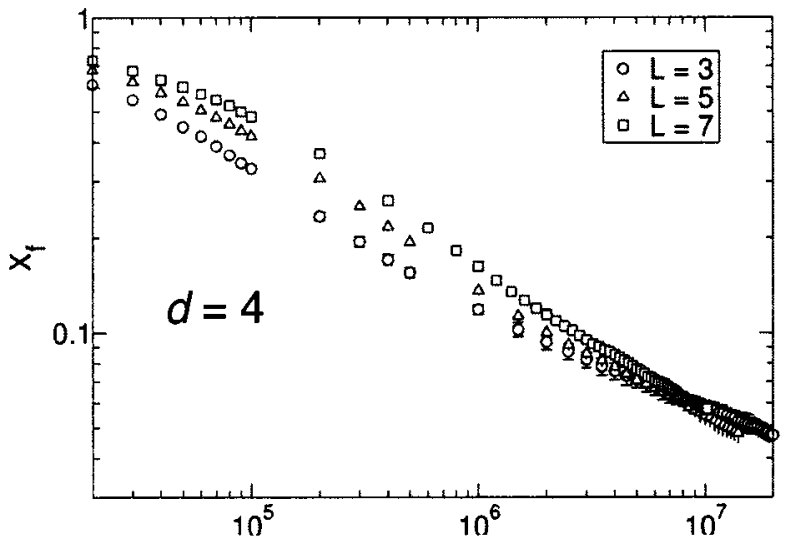

a

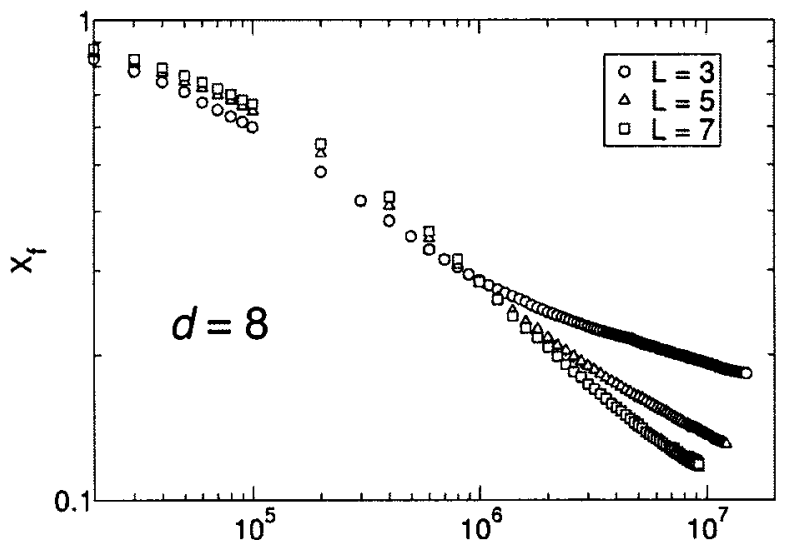

b

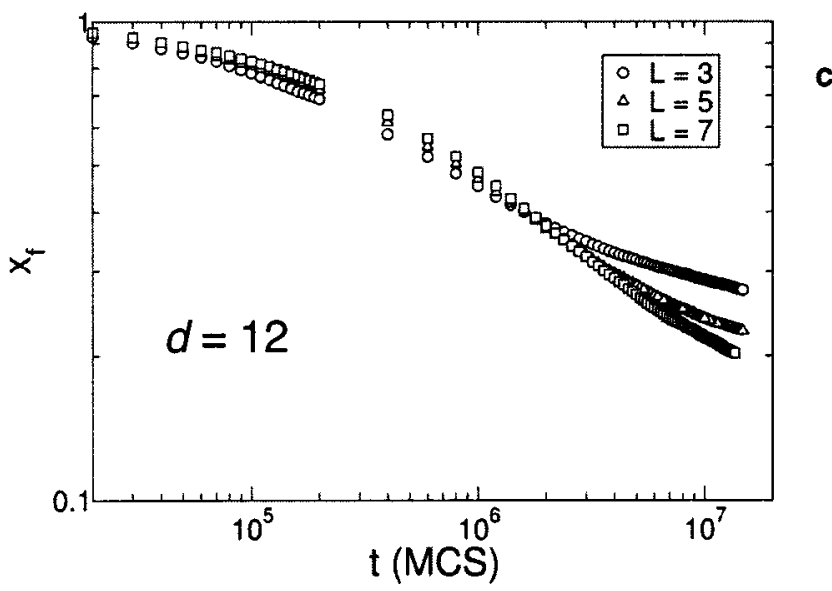

FIG. 2. Fraction of unreacted linker ends vs MCS as a function of linker length $L$ for NBBs of diameter (a) $d=4$, (b) $d=8$, (c) $d=12$. The data shown are the average of three independent runs and start from $t=10^{4}$. Error bars are shown when larger than the height of the symbol.

NBB functional sites and linker ends is largest for the smallest NBB $(d=4)$ due to the larger concentration of linker ends and NBB reactive sites.

\section{B. Rate of NBB-linker aggregate formation}

The fraction of unreacted linker ends characterizes the extent of reaction between NBBs and linkers, but this value alone cannot characterize the progress of the aggregation or the structural properties and size distribution of aggregates. To assess the rate of aggregate formation, ${ }^{52-54}$ we monitor the number of aggregates, $N(t)$, present in the system at a given time. Figures 3(a)-3(c) shows a plot of $N(t)$ versus $t$ on a double-logarithmic scale for the systems with linkers of 

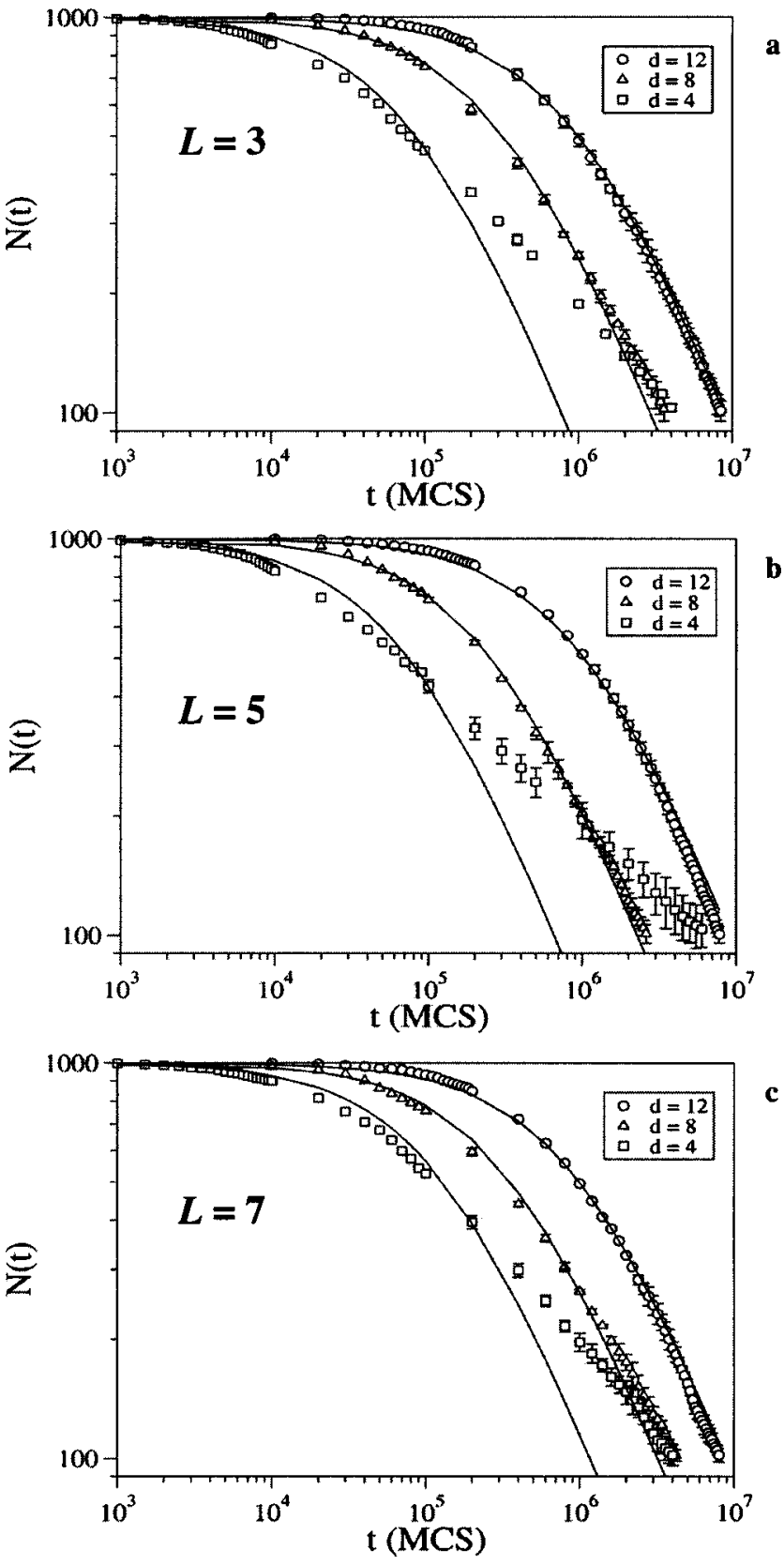

FIG. 3. Number of aggregates vs MCS as a function of NBB diameter $d$ for linker lengths (a) $L=3$, (b) $L=5$, (c) $L=7$. The data shown are the average of three independent runs. Error bars are shown when larger than the height of the symbol. Solid lines represent fits according to the Smoluchowski equation with a constant rate kernel in Eq. (2).

length $L=3,5$, and 7, and NBBs of diameter $d=4,8$, and 12. The data shown in Fig. 3 are the average of three independent runs; error bars are shown when the standard deviation is larger than the size of the symbol. Statistical noise is inherent in all Monte Carlo problems, and large systems as well as multiple independent runs are required to reduce the statistical error in calculated quantities. In our simulations, one typical run usually takes about 1 week to run 10 million MCS on an AMD Athlon 1600MP CPU. To complete the study in a reasonable amount of CPU time we conducted three independent runs for every system. The outcome is assuring because the statistical errors are not large, as shown in Fig. 3. Since the statistics become poorer at late stages of aggregation, to further counter the effects of statistical noise we neglect data collected when the total number of aggregates falls below $N(t)=100$ in Fig. 3 .

In Fig. 3, the scaling behavior of the kinetics of aggregation changes at an intermediate time, a trait that has also been observed in Brownian dynamics simulation of linear chain growth. ${ }^{55}$ Prior to the intermediate time, NBB aggregation is fast and after some intermediate time, the NBB aggregation kinetics follows a power law. Experimental data from the assembly of nanoparticles linked with streptavidin $^{56,57}$ suggests that the aggregation in biomoleculelinked nanoparticle systems may follow Smoluchowski ${ }^{53,58,59}$ kinetics, or a variant thereof. We first check to see if the aggregation behavior observed in our model systems follows the classical Smoluchowski model for colloidal aggregation. ${ }^{60}$ Smoluchowski theory consists of a set of differential equations that describe the rate of change of the number of clusters, $n_{s}$, containing $s$ particles during aggregation

$$
\frac{d n_{s}}{d t}=\frac{1}{2} \sum_{r=1}^{s-1} K_{r, s-r} n_{r} n_{s-r}-n_{s} \sum_{r=1}^{\infty} K_{s, r} n_{r} .
$$

The first term gives the rate of formation of clusters of size $s$ by the aggregation of two clusters of size $r$ and $s-r$. The second term gives the rate at which clusters of size $s$ are eliminated by aggregation with a cluster of size $r$. The population balance in Eq. (1) assumes that the collisions are binary and occur randomly, which is valid if the concentration of particles is sufficiently dilute that collisions between more than two clusters may be neglected. For Brownian aggregation of similarly sized, compact objects, the aggregation kernel, $K_{s, r}$, reduces to a constant, $k_{S}$ and Eq. (1) can then be solved analytically to give the following relationship between the total number of aggregates and time ${ }^{52}$

$$
N(t)=\frac{N(0)}{1+k_{S} N(0) t},
$$

where $N(t)$ is the total number of aggregates at time, $t, N(0)$ is the initial number of aggregates (here, NBBs) in the system, and $k_{S}$ is the aggregation rate constant. The solid lines in Fig. 3 show Eq. (2) fit to the simulation data. The aggregation data for the systems with $d=12$ agree well with the constant-kernel Smoluchowski model, with rate constants, $k_{S}=1.00 \pm 0.03 \times 10^{-9}, 1.05 \pm 0.04 \times 10^{-9}$, and $1.04 \pm 0.02$ $\times 10^{-9}$ for linkers of length $L=3,5$, and 7 , respectively. Thus, within statistical errors, the rate kernel for the $d=12$ systems is the same for all three linker lengths. The aggregation data for $d=8$ are also described well by the constantkernel Smoluchowski model, with rate constants, $k_{S}=3.77$ $\pm 0.08 \times 10^{-9}, 2.9 \pm 0.1 \times 10^{-9}$, and $2.63 \pm 0.07 \times 10^{-9}$ for linkers of length, $L=3,5$, and 7 , respectively, although at later times the model overpredicts the aggregation rate somewhat, most notably for the $d=8, L=7$ system. For systems with $d=4$, the aggregation data do not follow constantkernel Smoluchowski kinetics.

To understand why the constant-kernel Smoluchowski model applies to some NBB-linker systems but not others, it is helpful to consider the linker length to NBB size ratio, $\alpha$, 
TABLE I. Fractal dimensionality and the fraction of NBB pairs connected by four or more linkers.

\begin{tabular}{ccccc}
\hline \hline $\begin{array}{c}\text { NBB } \\
\text { diameter, } d \\
\text { (lattice } \\
\text { vertices) }\end{array}$ & $\begin{array}{c}\text { Monomers in } \\
\text { linker, } L\end{array}$ & $\begin{array}{c}\text { Linker to } \\
\text { NBB size } \\
\text { ratio, } \alpha\end{array}$ & $\begin{array}{c}\text { Fractal } \\
\text { dimension } \\
\text { estimated } \\
\text { from } g(r)\end{array}$ & $\begin{array}{c}\text { Fraction of NBB } \\
\text { pairs connected } \\
\text { by four or more } \\
\text { linkers }\end{array}$ \\
\hline 4 & 3 & 1.33 & 1.56 & 0.17 \\
4 & 5 & 2.00 & 1.74 & 0.11 \\
4 & 7 & 2.67 & 1.74 & 0.14 \\
8 & 3 & 0.57 & 1.4 & $4.3 e-3$ \\
8 & 5 & 0.85 & 1.48 & $2.5 e-2$ \\
8 & 7 & 1.14 & 1.51 & $5.0 e-2$ \\
12 & 3 & 0.36 & 1.47 & 0.0 \\
12 & 5 & 0.55 & 1.53 & 0.0 \\
12 & 7 & 0.73 & 1.49 & 0.0 \\
\hline \hline
\end{tabular}

obtained by dividing the average end-to-end distance of a linker, $\langle l\rangle$ (in units of lattice spacing) by the diameter of a NBB, $d-1$, measured in units of the lattice spacing. Table I shows the values of $\alpha$ for each system studied. For $\alpha<1$, the linkers are small enough such that their only role is to provide a strong interaction between NBBs at specific surface locations. In this case, the model system can be considered nearly equivalent to the simple model used by Smoluchowski for the coagulation of spherical particles. But for $\alpha>1$ the size disparity between NBBs and linkers decreases and the linkers contribute steric hindrance during aggregation which leads to significant deviation from the classical Smoluchowski aggregation model.

At long times, the asymptotic behavior for all systems is expected to follow a relation of the form ${ }^{61}$

$$
N(t) \propto t^{-\gamma},
$$

where $\gamma=1$ for the constant-kernel Smoluchowski model. Figure 4 shows a plot of $N(t)$ versus $t$ on a logarithmic scale for NBBs of diameter $d=4,8$, and 12 with linker length $L$ $=3$. Here, single run simulation data for time scales up to $10^{8}$ are used. A fit of the long time simulation data to the power law relationship in Eq. (3) gives the exponential fac-

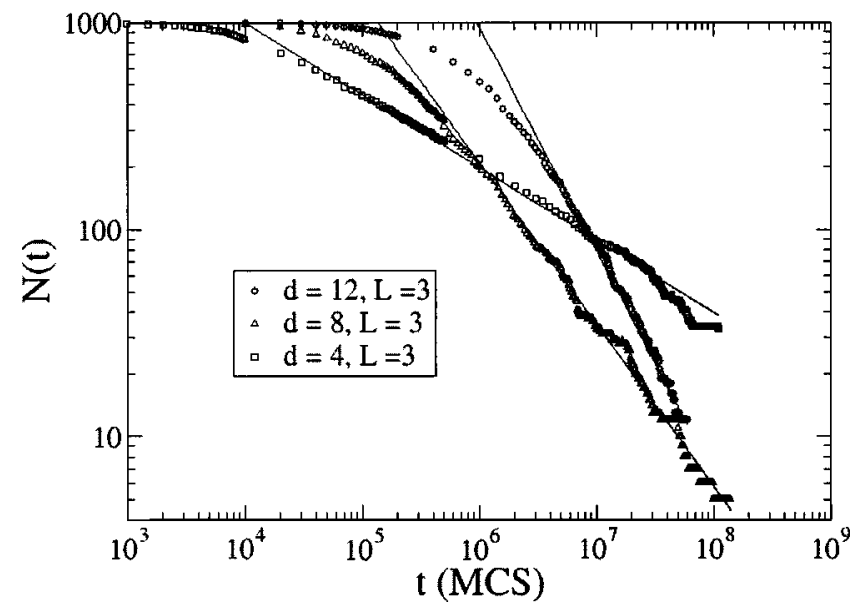

FIG. 4. Number of aggregates vs MCS as a function of NBB diameter $d$ for linker length $L=3$. The data shown contains only single-run simulation data. Solid lines represent fits of long-time simulation data according to the power law relationship in Eq. (3). tors $\gamma=0.41,0.83$ and 1.03 for $d=4,8$, and 12 , respectively, when $L=3$. The fact that $\gamma=1.03$ for the $d=12, L=3$ system provides further evidence that the classical, constantkernel Smoluchowski model describes the assembly behavior of the $d=12$ systems well. We have attempted to modify the model to obtain a better fit to the data with $\alpha>1$ or for the late stages of aggregation by employing a variable rate constant dependent on the fraction of available NBB surface binding sites, $\theta$ [e.g., $k_{\mathrm{S}} \propto \theta(1-\theta)$ (Refs. 56 and 57)] but are unable to find a universal model to describe the complete time behavior.

Another explanation for the disparity between the constant-kernel Smoluchowski model and the lattice MC simulation data for systems when $\alpha>1$ is the possibility that these systems transition from diffusion limited clustercluster aggregation (DLCA) to reaction limited clustercluster aggregation (RLCA). We have observed the rapid depletion of reactive sites on NBBs and linker ends (which are necessary for the further formation of larger aggregates) in $d=4$ systems. As a result, collision efficiency, which is directly related to the aggregation kernel or reaction probability, decreases significantly as the aggregation process proceeds. The higher fractal dimensionality of the $d=4$ systems (Table I) lends support to this possibility.

\section{Structure of the nanoparticle assemblies}

To understand the differences in the assembly process for the $d=4$ and $d=12$ NBBs we calculate several structural quantities to characterize the aggregates. First, we measure the spatial distribution of NBBs in the assembled aggregates by computing the pair correlation function, $g(r)$, of the NBBs. Figure 5 shows $g(r)$ for the aggregates, calculated using the center of the NBBs. The left column has data plotted according to NBB diameter; the right column has the same data plotted according to linker length. Figure 5(a) shows $g(r)$ for aggregates with a NBB of diameter $d=4$ and linkers of length $L=3,5$, and 7. Each $g(r)$ curve displays a first neighbor peak characteristic of structure with shortrange order. Figure 5(b) shows $g(r)$ for aggregates with a NBB of diameter $d=8$ and linkers of length $L=3,5$, and 7 . The correlation functions for $L=3$ and 5 display first and second neighbor peaks, indicating the structures are developing intermediate-range order. Figure 5(b) shows $g(r)$ for aggregates with a NBB of diameter $d=12$ and linkers of length $L=3,5$, and 7 . Here all correlation functions display first and second neighbor peaks where the second peak in the $L$ $=5$ and 7 data sets are more pronounced. Looking at Figs. $5(d)-5(f)$ in the right-hand column we see that NBB and linker combinations of $d=8$ and $L=3, d=12$ and $L=5$, and $d=12$ and $L=7$ lead to structures with higher order.

To explain why these particular combinations lead to higher-order structures we have developed an analysis code, TINGFO, described in the Appendix, to examine the structural topology of the aggregates. A bridge is formed when one linker connects two NBBs and one NBB pair can have multiple bridges between them. Table I lists the fraction of NBB pairs connected by four or more linkers. In the systems displaying a second peak in $g(r)$ we find that less than $0.5 \%$ of NBB pairs are connected by four or more linkers. In con- 

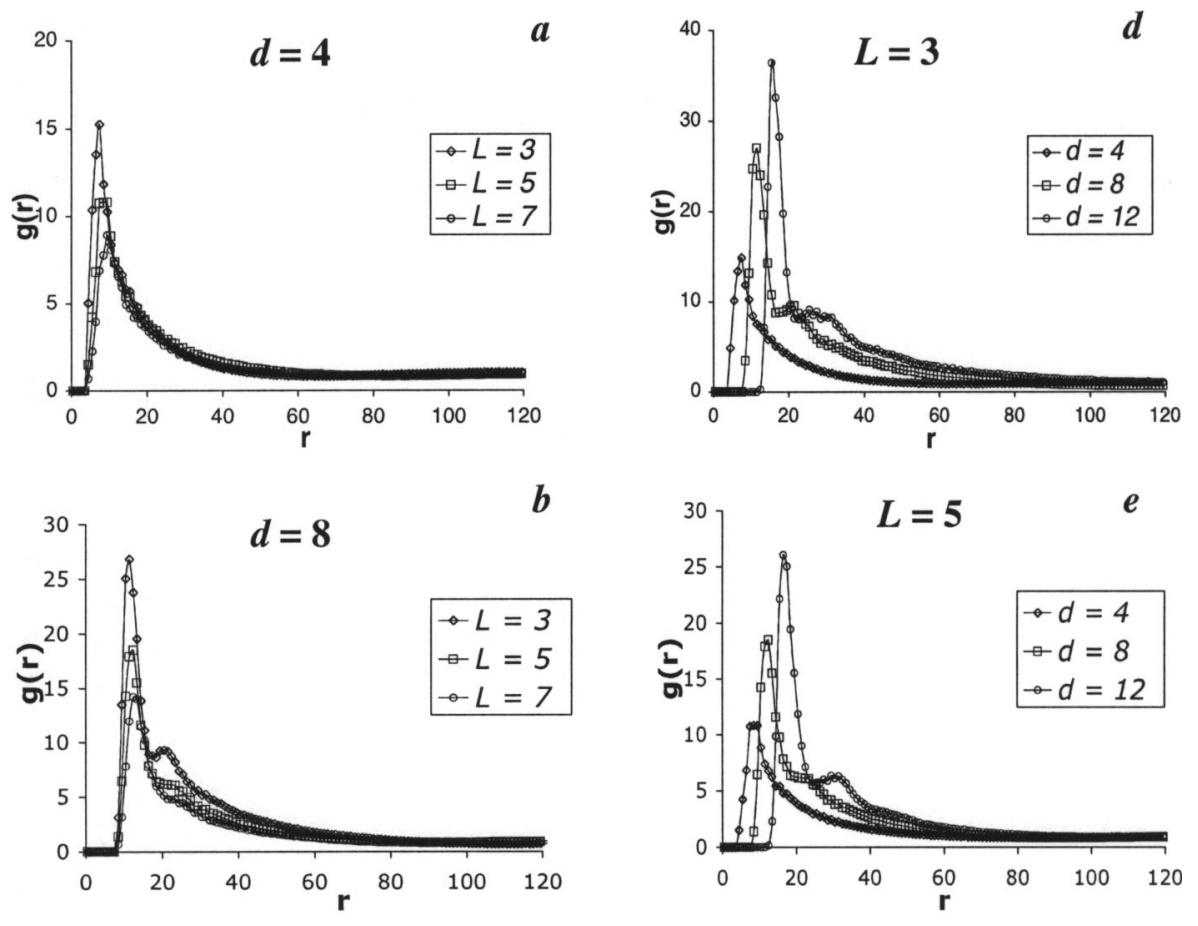

FIG. 5. Pair correlation function for NBBs in the linked aggregates shown as a function of linker length $L$ at various NBB diameters $d$ : (a) $d=4$, (b) $d$ $=8$, (c) $d=12$, and as a function of NBB diameter $d$ at various linker lengths $L$ [same data as in (a)-(c)]: (d) $L=3$, (e) $L=5$, (f) $L=7$.
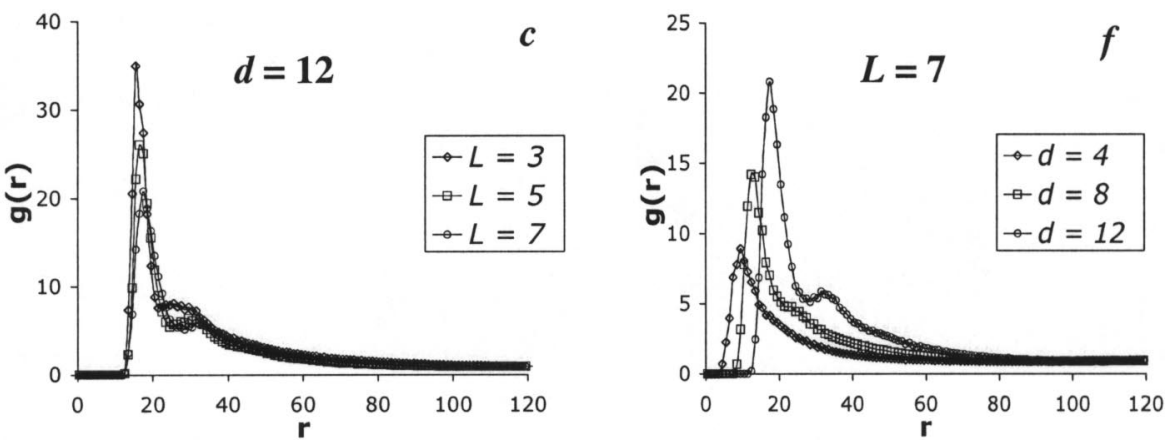

trast, for the systems without a second peak we find that $5-15 \%$ of the linked NBB pairs are connected by four or more linkers. Figure 6 shows snapshots of a representative aggregate from several systems with $d=4,8$, and 12 . The left column contains aggregates with linker length $L=3$; the right column contains aggregates with linker length $L=7$. Only linkers connecting two nanoparticles (i.e., bridges) are shown. The NBBs in the snapshots are not drawn to scale.

The systems with $d=8$ and $d=12$ form aggregates faster than the $d=4$ systems. Because linkers bind to the surface of the $d=4 \mathrm{NBBs}$ more easily, usually more than one linker will connect the same pair of NBBs. The formation of multiple-linker bridges between NBBs in the early stages of aggregation contributes steric hindrance and prevents linkers attached to other NBBs from binding to these pairs to form larger, extended aggregates. Figure 7 shows snapshots for the $d=4$ and $L=3$ system at 0.5 million MCS and the $d=12$ and $L=3$ system at 0.8 million MCS. In the $d=4$ system [Fig. 7(a)] there are 392 pairs of NBBs connected by multiple bridges of three or greater. Because the linker length is comparable to or even larger than the NBB size, the free reactive sites on the NBBs in these small aggregates are blocked by linkers within the aggregate [Fig. 7(b)]. This effectively decreases the probability of the small aggregates being joined to another NBB by a free linker end to form a bigger aggregate. In contrast, for the $d=12$ system [Fig. 7(c)] there are no NBBs connected by multiple bridges of three or greater. Here, the NBBs in the small aggregates are more "exposed," and thus, have a better chance to be linked with other NBBs to form bigger aggregates. Compact aggregates formed in $d=4$ systems and the open structure of aggregates formed in $d=12$ systems are also shown in Fig. 8 , which presents snapshots of aggregate formation at different times for $d=4, L=3$ [Figs. 8(a)-8(c)], and $d=12, L$ $=3$ [Figs. $8(\mathrm{~d})-8(\mathrm{f})]$.

\section{CONCLUSIONS}

Using Monte Carlo simulations of a minimal lattice model for nanoparticles and complementary biomolecule linkers we have shown that their aggregation may be described by classical Smoluchowski theory with a constant rate kernel when the linker length is shorter than the diameter of the nanoparticle $(\alpha<1)$. It is known that predictions by Smoluchowski models can describe many aggregation processes well and previous experimental work has shown the Smoluchowski theory or its variant is applicable to streptavidin-linked nanoparticle assembly, ${ }^{56,57}$ but the 


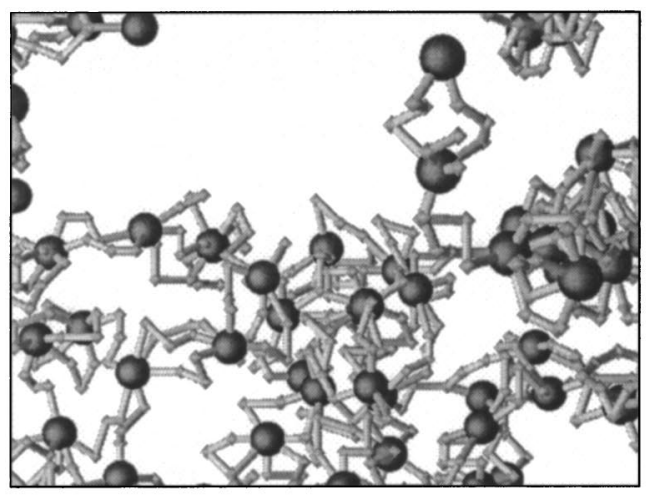

(a)

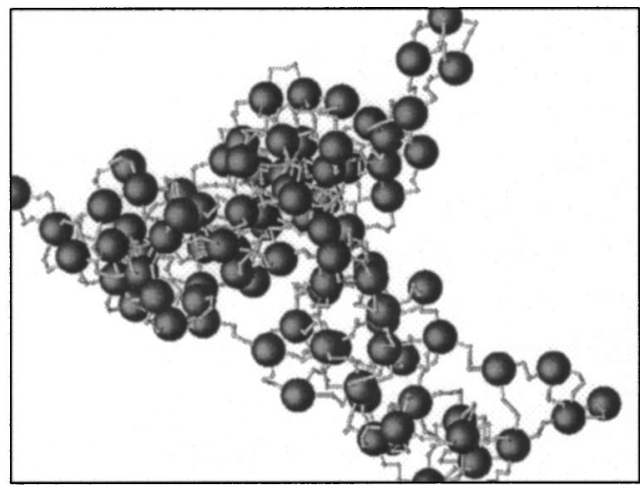

(b)

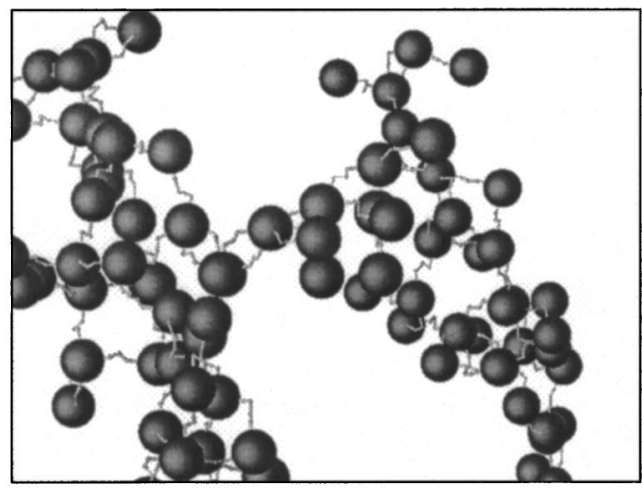

(c)

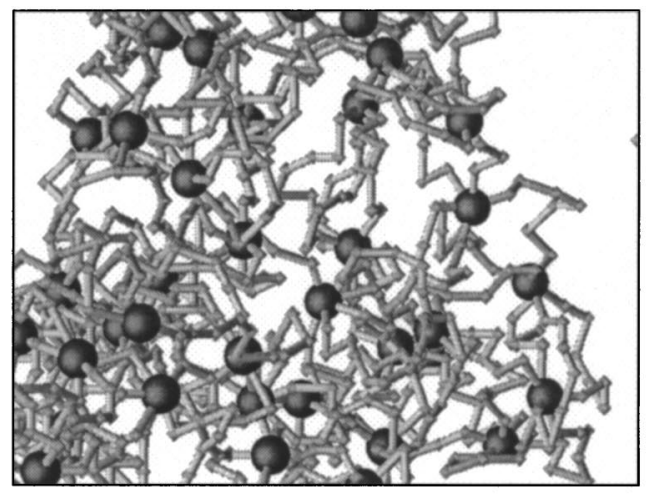

(d)

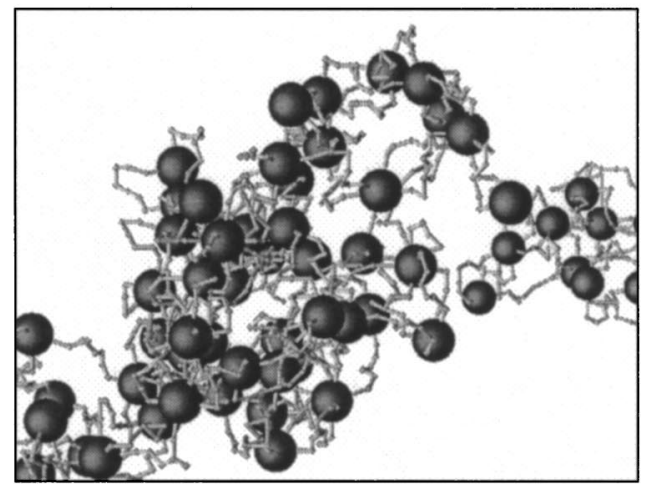

(e)

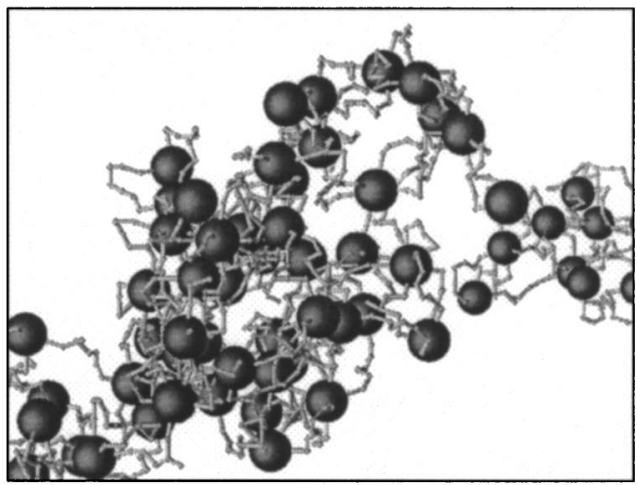

$(f)$

FIG. 6. Snapshots of aggregates with linker length $L=3$ : (a) $d=4$, (b) $d=8$, (c) $d=12$, and with linker length $L=7$ : (d) $d=4$, (e) $d=8$, (f) $d=12$. Only linkers connecting two nanoparticles (bridges) are shown. For clarity, the nanoparticles are not rendered to scale between images.

Smoluchowski theory has not been previously tested for a generalized model of biomolecule-linked nanoparticle assembly.

Our simulation results indicate that a generalized Smoluchowski theory with variable rate kernel may be a good starting point for building a predictive theory for biomoleculedirected assembly. Possible forms for the variable kernel may include $K_{r s}=r+s,{ }^{62} K_{r s}=r s,{ }^{63,64}$ or $K_{r s}=r^{\mu} s^{\nu}+r^{\nu} s^{\mu}$ (where $\mu$ and $\nu$ are variable exponents whose values depend on the aggregation process). ${ }^{65}$ Finding a suitable functional form of $K_{r s}$ for this model requires substantial input outside the scope of the present work. The first step in determining $K_{r s}$ is to calculate the diffusion coefficient for single clusters of size $n$ in the dilute solution limit. In NBB-linker systems, clusters with the same size $n$ may contain different numbers of linkers and/or have different topology (intra-cluster connectivity). Thus, in order to obtain the diffusion coefficient of a single cluster of size $n$, one must average over the different topologies available to each $n$, with the appropriate probabilities for each topology depending on the assembly process. While it is possible to carry out this calculation when $n$ is small, the task of averaging becomes prohibitive as $n$ increases, and is left for future studies.

\section{ACKNOWLEDGMENTS}

We thank Professor Robert Ziff for a critical review of this paper and many useful comments from which this paper 


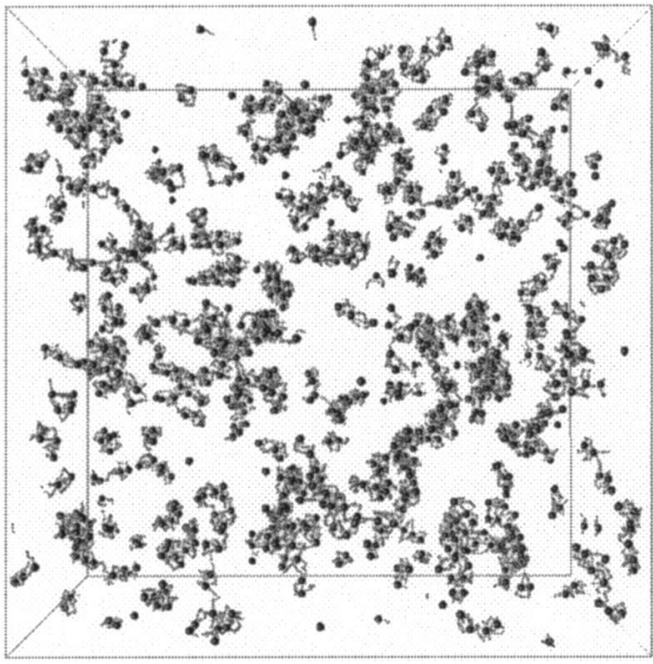

(a)

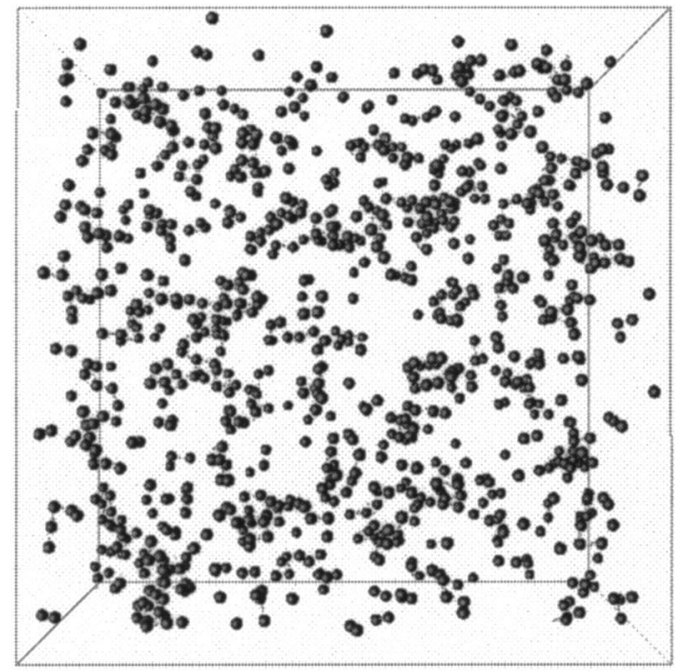

(c)

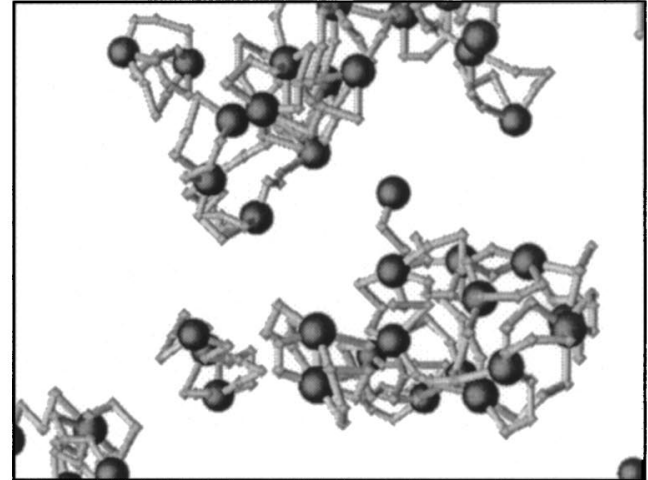

(b)

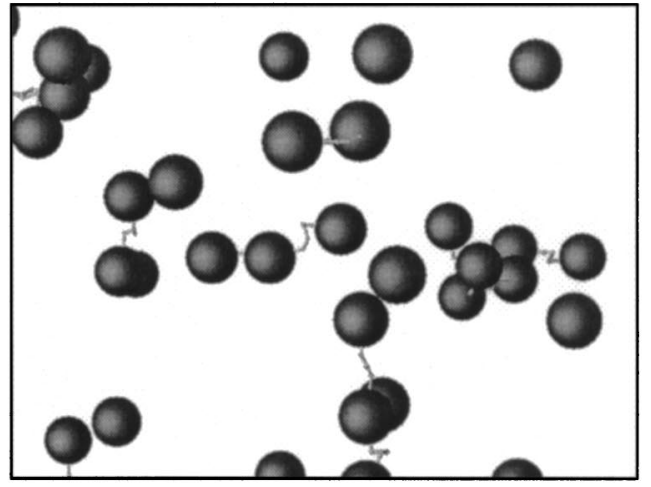

(d)

FIG. 7. Early time aggregation for (a) $d=4, L=3$ at 0.5 million MCS. (b) Close-up of an aggregate in (a) shows that several NBBs are connected by multiple linkers. Early time aggregation for (c) $d=12, L=3$ at 0.8 million MCS. (d) Close-up of an aggregate in (c) shows that no NBBs are connected by multiple linkers. For clarity, the nanoparticles are not rendered to scale between images.

has benefited. We thank Z. L. Zhang and M. A. Horsch for helpful discussions. Financial support for this work has been provided by the National Science Foundation, under Grant No. CTS-0210551-NER. We thank the National Partnership for Advanced Computational Infrastructure (NPACI) and the University of Michigan Center for Advanced Computing for a generous allocation of CPU time on the AMD-Athlon cluster.

\section{APPENDIX: ANALYSIS OF AGGREGATE PROPERTIES}

We developed an analysis code named TINGFO to determine structural properties such as elastic material fraction, fraction of defects in the aggregate, etc., by tracing and analyzing the interconnected linkers and NBBs within the resulting aggregates in the simulation. TINGFO can be used for both lattice and continuum models. The algorithm is similar in spirit to the SPANFO algorithm ${ }^{66}$ developed for analysis of graphical connectivity. TINGFO includes a routine to determine pendent material, the main defect in the aggregate. Similar analyses may be found in the work of Gilra et al. on end-linked polymer network properties. ${ }^{43}$

TINGFO performs the analysis with two procedures. The first step involves identifying aggregates in the system. Since we record the connectivity information for all NBBs and linker ends in the simulation through neighbor lists, we can easily identify to which NBB the current linker end under consideration is connected. We first treat every NBB as an aggregate of size one and label each aggregate with the serial number of the NBB it contains. Next, we loop through all linker ends sequentially and check for connections. When we find a linker connected to two NBBs (one NBB at each linker end), this linker is considered a bridge. We next check if either of the two NBBs have already been assigned to an aggregate with size greater than 2 . If not, the two linked 


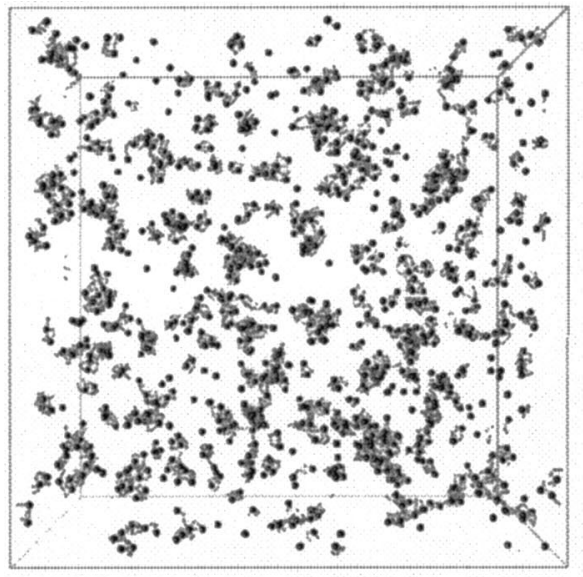

(a)

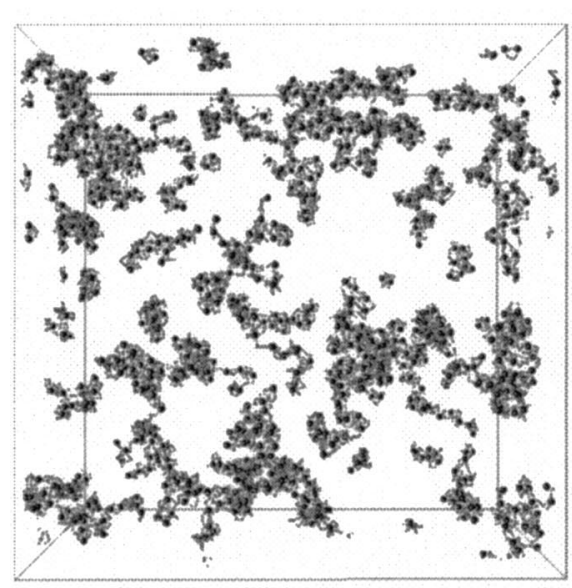

(b)

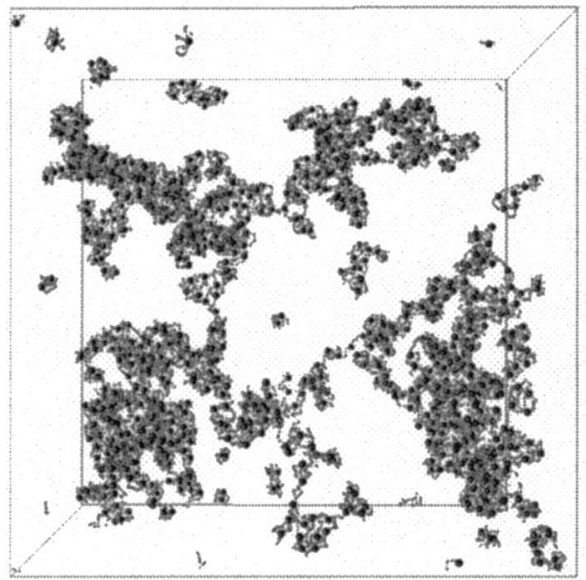

(c)

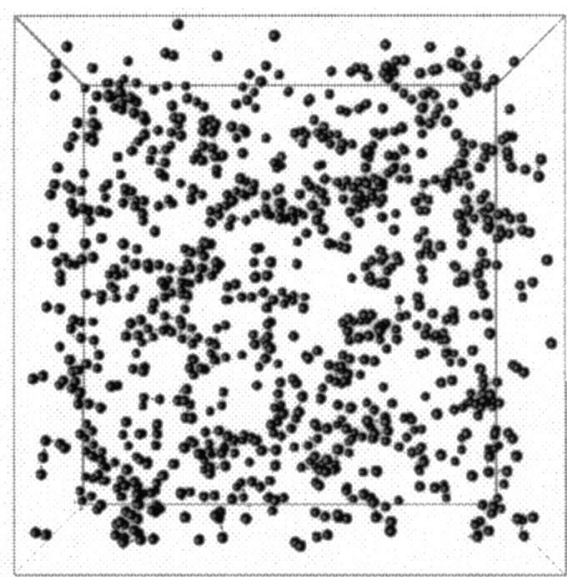

(d)

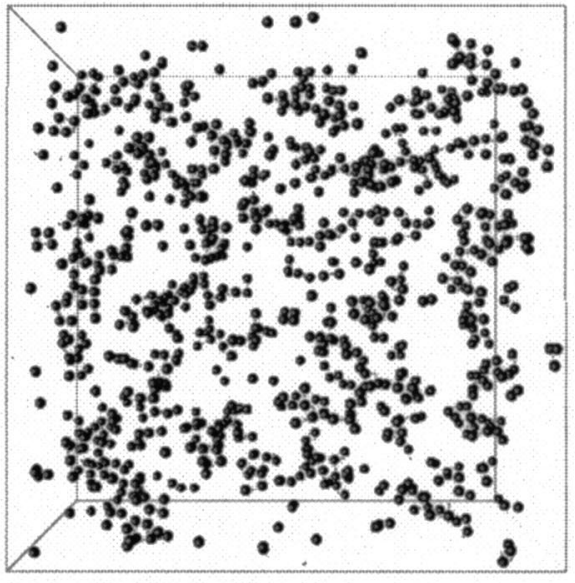

(e)

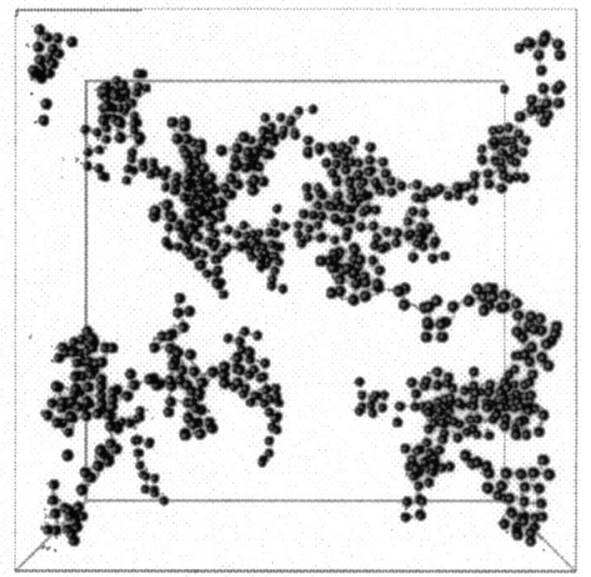

$(f)$
FIG. 8. Time evolution of aggregate formation for $d=4, L=3$ : (a) 0.1 million MCS, (b) 7 million MCS, (c) 60 million MCS, and for $d=12, L=3$ : (d) 0.8 million MCS, (e) 1.4 million MCS, (f) 60 million MCS. For clarity, the nanoparticles are not rendered to scale between images.
NBBs are considered an aggregate and the aggregate will be labeled with the smaller of the serial numbers identifying the two NBBs. If one or both of the linked NBBs belong to aggregates with size greater than 2 , then the two aggregates or one NBB and one aggregate will be considered to form a new aggregate with the label of the smaller aggregate and a size corresponding to the sum of their sizes before their combination into one aggregate. After looping through all the linker ends, we can identify all aggregates in a single round, with their size given in terms of the number of NBBs in the aggregate. Once a NBB or aggregate is assigned to a bigger aggregate, all linkers on that NBB or aggregate are considered part of the bigger aggregate.

While the method used to loop through connecting linkers and identify aggregates may not be as efficient as the SPANFO algorithm, it is very straightforward to implement. Since the time used to identify all aggregates is typically on the order of seconds even for a large system, ${ }^{50}$ the cost for post-simulation analysis is negligible relative to the simulation CPU time, which is typically on the order of days or weeks.

The second procedure in TINGFO involves determining 
the structural properties of the largest aggregate, i.e., the network. The aggregate containing the largest number of NBBs, which is considered to be the network, usually also contains the largest number of linkers. All remaining material including smaller aggregates, free NBBs, and free linkers are regarded as soluble material. We define the following relationship: elastic material fraction $=1-\{$ soluble fraction $\}$ $-\{$ fraction of loops $\}-\{$ fraction of pendent material $\}$. The soluble fraction is computed as

$$
w_{\mathrm{sol}}=1-\frac{n_{1} L S_{1}+m_{1} S_{2}}{N L S_{1}+M S_{2}},
$$

where $n_{1}$ and $m_{1}$ are the number of linkers and NBBs, respectively, in the network, $N$ and $M$ are the total number of linkers and NBBs, respectively, in the system, $L$ is the length of a linker, and $S_{1}=2 \times 2 \times 2$ and $S_{2}=d \times d \times d$ are the size of a monomer in a linker and a NBB, respectively. This definition of soluble fraction is identical to the one used by Gilra et al. in the study of end-linked polymer networks. ${ }^{43}$

Unlike the definition used by Gilra et al., we do not differentiate between the single chain loops in the pendent material and the single chain loops attached to the elastic network. Instead, we combine these two definitions into one parameter termed fraction of loops, which includes all single chain loops in the network. Any loop containing more than one chain is not included. Also, when calculating the fraction of loops, we first exclude the loops contained in the soluble material to avoid double counting. The fraction of loops can be computed as

$$
w_{\text {loops }}=\frac{n_{2} L S_{1}}{N L S_{1}+M S_{2}},
$$

where $n_{2}$ is the number of single chain loops. Note that in the model considered in this work, each linker end has its own specific interaction to one of two types of NBBs in the system, and thus, the formation of loops is prohibited.

Determining the fraction of pendent material is the most difficult part of characterizing the network's structural properties. The pendent material includes dangling chains, dangling NBBs, and any structure with only a single path to the network. The procedure of identifying the pendent material in TINGFO is similar to the "burning algorithm" 67 often encountered in percolation theory and digital-image-based simulations. To check continuity from the outer side of the network to the inner side of the network, for example, all the terminal ends are regarded as combustible. Terminal ends along the outer edge are "set on fire," and the "fire" is allowed to propagate inward until no more terminal ends can be burned. We use the following procedure to define terminal ends. An unlinked linker end (called a dangling chain) is considered a terminal end, and thus the whole linker with only one end linked to the network will be burned entirely, as illustrated in Fig. 9. ${ }^{68}$

After these dangling chains are burned, a new round of finding terminal ends commences. Now, the following three cases illustrated in Fig. 9 are considered: single bridge NBBs, double bridge NBBs and triple bridge NBBs. ${ }^{69}$ We then burn these terminal ends, as well as the bridges between terminal ends and the network. We repeat this procedure un-
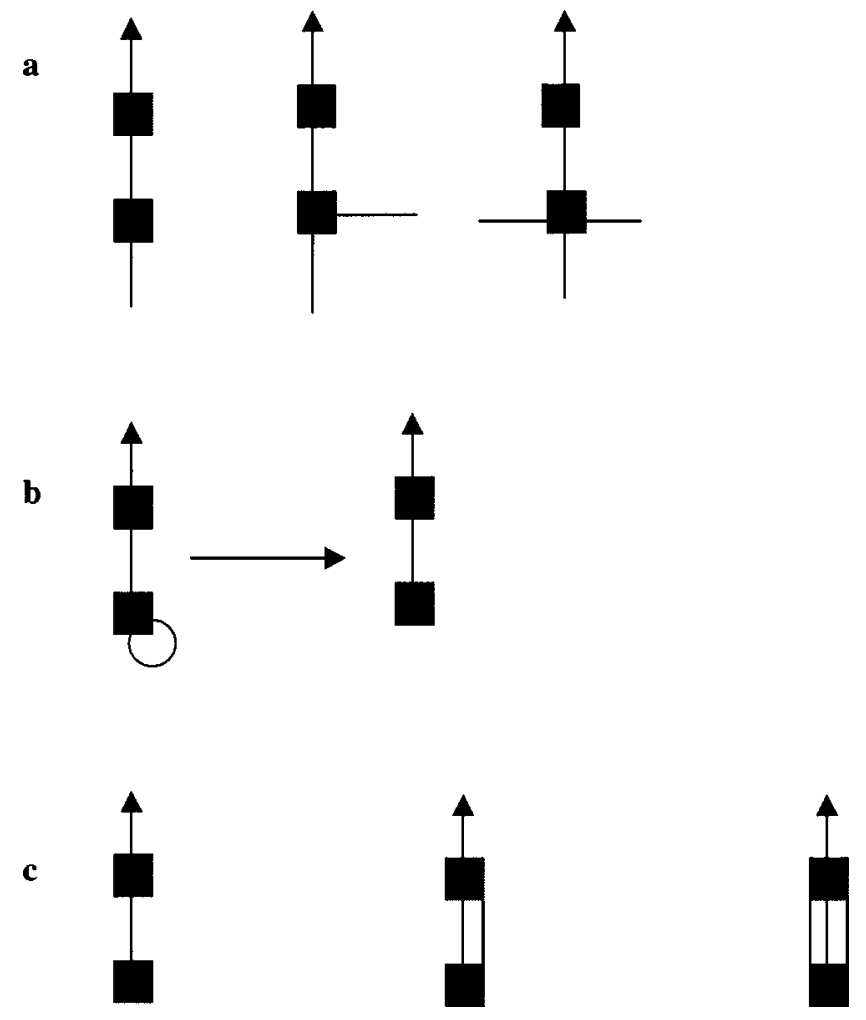

FIG. 9. Pendent structures: (a) dangling linker ends, (b) single linker loop, (c) terminal NBBs. The arrow indicates the connection to the aggregate or network.

til we reach a state where no terminal ends remain according to the previously described definition. The fraction of pendent material is then computed as:

$$
w_{\text {pendent }}=\frac{\left(n_{3}+n_{4}\right) L S_{1}+m_{2} S_{2}}{N L S_{1}+M S_{2}},
$$

where $n_{3}$ and $n_{4}$ are the number of dangling chains and bridges burned in the routine, respectively; $m_{2}$ is the number of NBBs that are burned as terminal ends in the routine. Finally, after subtracting all the above defects from the network the remaining NBBs and linkers comprise the network's elastic material.

Currently we have utilized only part of the information provided by TINGFO, i.e., information on the aggregate numbers, sizes, and topology. We will use the algorithm to calculate additional network properties when we simulate volume fractions above the percolation threshold where properties such as fraction of elastic material and fraction of pendent material become important. These properties will be addressed in a forthcoming paper.

${ }^{1}$ C. J. Murphy, Science 298, 2139 (2002).

${ }^{2}$ R. D. Kamien, Science 299, 1671 (2003).

${ }^{3}$ W. U. Huynh, J. J. Dittmer, and A. P. Alivisatos, Science 295, 2425 (2002).

${ }^{4}$ J. J. Urban, W. S. Yun, Q. Gu, and H. Park, J. Am. Chem. Soc. 124, 1186 (2002).

${ }^{5}$ Y. G. Sun and Y. N. Xia, Science 298, 2176 (2002).

${ }^{6}$ R. Jin, Y. Cao, C. A. Mirkin, K. L. Kelly, G. C. Schatz, and J. G. Zheng, Science 294, 1901 (2001).

${ }^{7}$ G. P. Mitchell, C. A. Mirkin, and R. L. Letsinger, J. Am. Chem. Soc. 121, 8122 (1999) 
${ }^{8}$ R. L. Letsinger, R. Elghanian, G. Viswanadham, and C. A. Mirkin, Bioconjugate Chem. 11, 289 (2000).

${ }^{9}$ L. Xu, Y. Guo, R. Xie, J. Zhuang, W. Yang, and T. Li, Nanotechnology 13, 725 (2002)

${ }^{10}$ J. Liu, J. Alvarez, and A. E. Kaifer, Adv. Mater. (Weinheim, Ger.) 12, 1381 (2000).

${ }^{11}$ E. Dujardin, L.-B. Hsin, C. R. C. Wang, and S. Mann, Chem. Commun. (Cambridge) 14, 1264 (2001).

${ }^{12}$ A. Star, J. F. Stoddart, D. Steuerman et al., Angew. Chem., Int. Ed. Engl. 40, 1721 (2001)

${ }^{13}$ R. J. Chen, Y. Zhang, D. Wang, and H. Dai, J. Am. Chem. Soc. 123, 3838 (2001).

${ }^{14}$ G. R. Dieckmann, A. B. Dalton, P. A. Johnson et al., J. Am. Chem. Soc. 125, 1770 (2003).

${ }^{15}$ C. A. Dyke and J. M. Tour, J. Am. Chem. Soc. 125, 1156 (2003).

${ }^{16}$ A. M. Cassell, W. A. Scrivens, and J. M. Tour, Angew. Chem., Int. Ed. Engl. 37, 1528 (1998).

${ }^{17}$ R. M. Laine, J. W. Choi, and I. Lee, Adv. Mater. (Weinheim, Ger.) 13, 800 (2001).

${ }^{18}$ R. O. R. Costa, W. L. Vasconcelos, R. Tamaki, and R. M. Laine, Macromolecules 34, 5398 (2001).

${ }^{19}$ J. Choi, J. Harcup, A. F. Yee, Q. Zhu, and R. M. Laine, J. Am. Chem. Soc. 123, 11420 (2001).

${ }^{20}$ C. X. Zhang, F. Babonneau, C. Bonhomme, R. M. Laine, C. L. Soles, H. A. Hristov, and A. F. Yee, J. Am. Chem. Soc. 120, 8380 (1998).

${ }^{21}$ C. A. Mirkin, R. L. Letsinger, R. C. Mucic, and J. J. Storhoff, Nature (London) 382, 607 (1996).

${ }^{22}$ S. Mann, W. Shenton, M. Li, S. Connolly, and D. Fitzmaurice, Adv. Mater. (Weinheim, Ger.) 12, 147 (2000).

${ }^{23}$ C. M. Niemeyer, Curr. Opin. Chem. Biol. 4, 609 (2000).

${ }^{24}$ A. K. Boal, F. Ilhan, J. E. DeRouchey, T. Thurn-Albrecht, T. P. Russell, and V. M. Rotello, Nature (London) 404, 746 (2000).

${ }^{25}$ B. L. Frankamp, O. Uzun, F. Ilhan, A. K. Boal, and V. M. Rotello, J. Am. Chem. Soc. 124, 892 (2002).

${ }^{26}$ A. K. Boal, M. Gray, F. Ilhan, G. M. Clavier, L. Kapitzky, and V. M. Rotello, Tetrahedron 58, 765 (2002).

${ }^{27}$ J. J. Storhoff, R. C. Mucic, and C. A. Mirkin, J. Cluster Sci. 8, 179 (1997).

${ }^{28}$ C. M. Niemeyer, Angew. Chem., Int. Ed. Engl. 40, 4128 (2001).

${ }^{29}$ R. C. Mucic, J. J. Storhoff, C. A. Mirkin, and R. L. Letsinger, J. Am. Chem. Soc. 120, 12674 (1998).

${ }^{30}$ K. J. Watson, S. J. Park, J. H. Im, S. T. Nguyen, and C. A. Mirkin, J. Am. Chem. Soc. 123, 5592 (2001)

${ }^{31}$ S. Connolly and D. Fitzmaurice, Adv. Mater. (Weinheim, Ger.) 11, 1202 (1999).

${ }^{32}$ M. Li, K. K. W. Wong, and S. Mann, Chem. Mater. 11, 23 (1999).

${ }^{33}$ W. Shenton, S. A. Davis, and S. Mann, Adv. Mater. (Weinheim, Ger.) 11, 449 (1999)

${ }^{34}$ J. J. Storhoff, A. A. Lazarides, R. C. Mucic, C. A. Mirkin, R. L. Letsinger, and G. C. Schatz, J. Am. Chem. Soc. 122, 4640 (2000).

${ }^{35}$ I. Carmesin and K. Kremer, Macromolecules 21, 2819 (1988).

${ }^{36}$ H. P. Deutsch and K. Binder, J. Chem. Phys. 94, 2294 (1991).

${ }^{37}$ W. Paul, K. Binder, D. W. Heermann, and K. Kremer, J. Chem. Phys. 95, 7726 (1991)

${ }^{38}$ A. Z. Panagiotopoulos and S. K. Kumar, Phys. Rev. Lett. 83, 2981 (1999).

${ }^{39}$ M. Schulz and J. U. Sommer, J. Chem. Phys. 96, 7102 (1992).

${ }^{40}$ M. Schulz and H. L. Frisch, J. Chem. Phys. 101, 10008 (1994).

${ }^{41}$ H. L. Trautenberg, J. U. Sommer, and D. Goritz, J. Chem. Soc., Faraday Trans. 91, 2649 (1995).
${ }^{42}$ C. Hagn, M. Wittkop, S. Kreitmeier, H. L. Trautenberg, T. Holzl, and D. Goritz, Polym. Gels Networks 5, 327 (1997).

${ }^{43}$ N. Gilra, C. Cohen, and A. Z. Panagiotopoulos, J. Chem. Phys. 112, 6910 (2000)

${ }^{44}$ W. Michalke, S. Kreitmeier, M. Lang, A. Buchner, and D. Goritz, Comput. Theor. Polym. Sci. 11, 459 (2001).

${ }^{45}$ W. Michalke, M. Lang, S. Kreitmeier, and D. Goritz, J. Chem. Phys. 117, 6300 (2002).

${ }^{46}$ Z. Chen, C. Cohen, and F. A. Escobedo, Macromolecules 35, 3296 (2002).

${ }^{47}$ L. M. Demers, C. A. Mirkin, R. C. Mucic, R. A. Reynolds, R. L. Letsinger, R. Elghanian, and G. Viswanadham, Anal. Chem. 72, 5535 (2000).

${ }^{48}$ L. Stryer, Biochemistry, 4th ed. (W. H. Freeman, New York, 1995).

${ }^{49}$ A. Di Cecca and J. Freire, Macromolecules 35, 2851 (2002).

${ }^{50}$ M. H. Lamm, T. Chen, and S. C. Glotzer, Nano Lett. 3, 989 (2003).

${ }^{51}$ Although time is not an inherent quantity in Monte Carlo simulations, in simulations employing the bond fluctuation algorithm, a dynamic Monte Carlo algorithm, one MCS may be considered proportional to a unit of time.

${ }^{52}$ A. A. Rzepiela, J. H. J. van Opheusden, and T. van Vliet, J. Colloid Interface Sci. 244, 43 (2001).

${ }^{53}$ R. M. Ziff, E. D. McGrady, and P. Meakin, J. Chem. Phys. 82, 5269 (1985).

${ }^{54}$ S. Salaniwal, S. T. Cui, H. D. Cochran, and P. T. Cummings, Langmuir 17, 1784 (2001).

${ }^{55}$ M. C. Buján-Núñez and B. Vázquez-Varela, Mol. Phys. 98, 1011 (2000).

${ }^{56}$ E. T. Kisak, M. T. Kennedy, D. Trommeshauser, and J. A. Zasadzinski, Langmuir 16, 2825 (2000).

${ }^{57}$ S. Connolly, S. Cobbe, and D. Fitzmaurice, J. Phys. Chem. B 105, 2222 (2001).

${ }^{58}$ M. Z. Smoluchowski, Z. Phys. Chem., Stoechiom. Verwandtschaftsl. 92, 129 (1917).

${ }^{59}$ P. Meakin, T. Vicsek, and F. Family, Phys. Rev. B 31, 564 (1985).

${ }^{60}$ S. K. Friedlander, Smoke, Dust and Haze: Fundamentals of Aerosol Behavior (Wiley, New York, 1977).

${ }^{61}$ J. Schmelzer, G. Ropke, and R. Mahnke, Aggregation Phenomena in Complex Systems (Wiley-VCH, Weinheim, 1999).

${ }^{62}$ W. T. Scott, J. Atmos. Sci. 25, 54 (1968).

${ }^{63}$ J. B. McLeod, Quarterly J. Math. 13, 119, 193 (1962).

${ }^{64}$ R. M. Ziff, J. Stat. Phys. 23, 241 (1980).

${ }^{65}$ P. G. J. van Dongen and M. H. Ernst, Phys. Rev. Lett. 54, 1396 (1985).

${ }^{66} \mathrm{~A}$. Nijenhuis and H. S. Wilf, Combinatorial Algorithms (Academic, New York, 1975)

${ }^{67} \mathrm{D}$. Stauffer and A. Aharony, Introduction to Percolation Theory, 2nd ed. (Taylor and Francis, London, 1994).

${ }^{68}$ By this definition, loops will not be considered as terminal ends. But since loops are already considered above, the code will also burn them, if there are any, as illustrated in Fig. 1(b).

${ }^{69}$ In this work, we use hexafunctional NBBs in which the functional sites are located in the center of each of six faces, so double-bridge and triplebridge NBBs illustrated in Fig. 1(c) are relatively rare unless the linker is long.

${ }^{70}$ Z. L. Zhang, M. A. Horsch, M. H. Lamm, and S. C. Glotzer, Nano Lett. 3, 1341 (2003).

${ }^{71}$ Z. L. Zhang and S. C. Glotzer, Nano Lett. (in press). 